\title{
Desenvolvimento de um agente SNMP para monitoramento de ambientes empresariais
}

\author{
Gerson Dalcin', Ricardo Becker ${ }^{1,2}$ \\ ${ }^{1}$ Universidade de Caxias do Sul \\ 95700-000 - Bento Gonçalves - RS - Brazil \\ ${ }^{2}$ Faculdade SENAI de Tecnologia \\ 91140-000 - Porto Alegre - RS - Brazil \\ gdalcin2@ucs.br, ricardobecker.eng@gmail.com
}

\begin{abstract}
Resumo. Conforme tendência da indústria 4.0, o monitoramento e busca de dados sobre máquinas está cada vez mais insurgente. As tecnologias IoT buscam auxiliar nas soluções em coleta de dados e facilitar a comunicação entre dispositivos de rede. O protocolo SNMP se caracteriza por ser robusto e de simples manuseio para a coleta de dados e monitoramento dos ativos. Este artigo apresenta a proposta de desenvolvimento de um agente SNMP modular para monitoramento a nível empresarial elou industrial.
\end{abstract}

\section{Introdução}

A tecnologia sempre foi um dos pilares dos avanços na produtividade e competividade no âmbito industrial. Com a mesma, foi possível alcançar os desenvolvimentos necessários para dar o início as revoluções industriais. Hoje, nos encontramos em meio a quarta revolução que se dá pelo avanço da Internet das Coisas (IoT - Internet of Things) em ambientes de fabricação. Sistemas de coletas, armazenagem e processamento de dados em máquinas são fundamentais para $\mathrm{o}$ desenvolvimento fabril, de modo a deixar as instalações ainda mais inteligentes e rentáveis [Schneider 2018].

Para obter uma rede IoT, é necessário possuir suporte para aplicações e serviços heterogêneos, isto envolve qualquer objeto que pode ser conectado tanto em redes locais, como globais. Nos dias atuais, há uma perspectiva de 50 bilhões de dispositivos conectados na internet e com um valor estimado de U\$7,1 trilhões [Silva et al. 2018].

O protocolo Simple Network Management Protocol (SNMP), é um protocolo não orientado a conexão, o próprio, é um protocolo simples e robusto e ao mesmo tempo, poderoso o suficiente para resolver problemas em gerenciamento de redes [Silva 2018]. O protocolo SNMP foi utilizado basicamente para controlar a disponibilidade e desempenho de dispositivos como roteadores, switches, servidores e outras interfaces que disponibilizavam o agente SNMP [Poloni et al. 2017].

O objetivo de desenvolvimento deste artigo é proposto através da criação de um agente SNMP microcontrolado, o sistema será modular e de fácil implementação em conjunto com o monitoramento ativo existente. Ele será ajustado para os vários aspectos industriais e empresariais encontrados. A prova de conceito, se dará em uma aplicação de monitoramento de ambientes de centro de processamento de dados (Data Center). 
Este artigo consiste em cinco seções, incluindo a introdução. Para a seção 2 é apresentado uma contextualização da indústria 4.0 na atualidade, enquanto na seção 3 , possui uma base teórica sobre o protocolo SNMP e suas principais operações. Na seção 4 é apresentado a proposta e metodologia a ser aplicada e por fim, é demonstrado os resultados esperados após a finalização da prática na seção 5.

\section{Indústria 4.0 e Internet das Coisas (IoT)}

O termo Indústria 4.0 ficou conhecido em 2011, quando uma junção público/privada alemã promoveu uma abordagem a fim de aprimorar a competitividade industrial nacional. Este movimento criou o que chamamos de fábricas inteligentes, onde possuem estruturas modulares em que sistemas cíber físicos monitoram processos e tomam decisões descentralizadas. Essa transformação se dá pela interconexão de sensores, máquinas, peças e sistemas de informação em uma cadeia de valores multi empresas. Esses sistemas denominados cíber físicos, podem interagir uns com os outros usando protocolos padrões e análise de dados para prever falhas e prover monitoramento nos ativos. Através desta análise, é possível organizar processos com mais rapidez, flexibilidade e eficiência, mantendo sempre a alta qualidade e diminuindo custos operacionais com o aumento da produtividade de manufatura [Schneider 2018]. A Figura 1, demonstra as etapas de cada evolução industrial até os dias atuais [Kagermann et al. 2013].

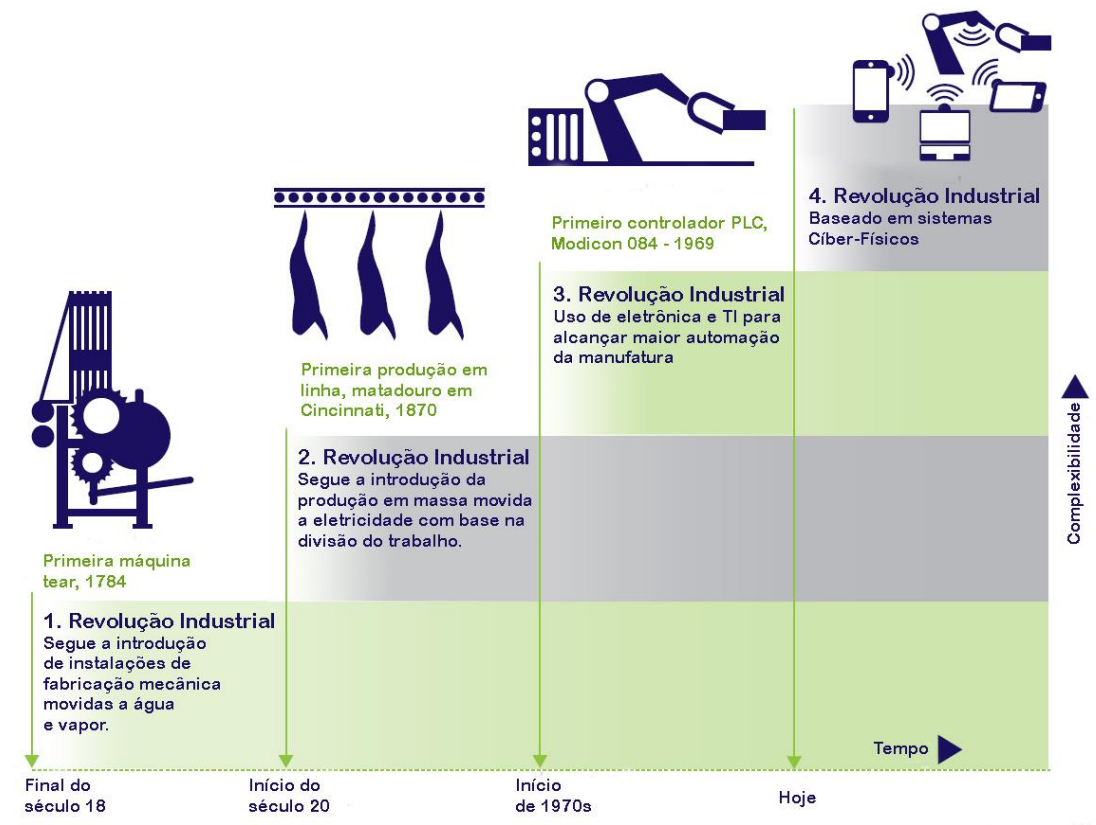

Figura 1. Revoluções Industriais [Kagermann et al. 2013] (Adaptado pelo Autor).

Um dos pilares da Indústria 4.0 é a Internet das Coisas. O conceito foi introduzido primeiramente em 1999, onde foi utilizado para nomear um sistema de comunicação entre sensores e computadores. Este conceito envolve a combinação de dispositivos identificáveis através de uma rede, esses objetos possuem a possibilidade de coleta, processamento ou troca de dados de forma direta ou indireta. A capacidade da IoT de integrar com as redes clássicas e objetos de rede, vem ao encontro com a construção de um ambiente inteligente que nos circula nos dias atuais [Schneider 2018]. 
Esses dispositivos são apresentados de forma direta e/ou indireta para a população, através de telefones, tags RFID (Radio Frequency Identification), entre outros. Para o objeto ser reconhecido como um dispositivo de IoT, o mesmo deve possuir um baixo consumo e poder computacional. Em estudos direcionados a saúde, é buscado a possibilidade de as pessoas possuírem um chip que efetue o monitoramento da saúde através de sensores na residência, e ser possível acionar uma ajuda médica caso necessário de forma autônoma [Poloni et al. 2017].

Desta forma, um dos desafios mais críticos para o gerenciamento IoT, é a interoperabilidade e heterogeneidade de comunicação dos protocolos com os dispositivos, onde se faz necessária uma rede de dispositivos que é capaz de extrair dados individualmente de cada objeto e intercomunicando-se entre si [Silva et al. 2018].

\section{Protocolo SNMP}

O SNMP apresenta-se como um protocolo da camada de aplicação que compõe a pilha do TCP/IP (Transmission Control Protocol / Internet Protocol), o mesmo é amplamente utilizado na gerência de redes e ativos. O SNMP foi aprovado pela Internet Architecture Board (IAB) em 1988, como o protocolo padrão para gerenciamento de redes IP, hoje o protocolo é o mais utilizado em gerenciamento de redes. O SNMP utiliza um protocolo de simples solicitação/resposta, que executa sobre o User Datagram Protocol (UDP), que está na camada de transporte da pilha TCP/IP. Com isso, minimizando a complexidade dos procedimentos de comunicação e implementação [Machado 2015][Sinche et al. 2020]. O SNMP baseia-se em arquitetura cliente/servidor e consiste nos seguintes componentes (Figura 2) [Sinche et al. 2020]:

a. Dispositivo gerenciado: um nó de rede onde um agente está localizado.

b. Agente: Software de gerenciamento de rede modular, responsável pela manutenção das informações dos dispositivos. O mesmo, recebe requisições provenientes do gerente e envia as informações relativas a requisição. Também é responsável por enviar alterações sobre condições anormais previamente cadastradas.

c. Gerente: Software de gerenciamento SNMP, normalmente instalado em servidores. O mesmo envia solicitações e recebe respostas / notificações dos agentes, sobre informações de dispositivos gerenciados, a fim de realizar tarefas. Possui comunicação com todos os agentes disponíveis na rede. É responsável pelo monitoramento, geração de relatórios e controles de falhas.

d. Sistema de gerenciamento de rede (NMS): monitora e controla dispositivos gerenciados usando aplicativos de gerenciamento.

e. Management Information Base (MIB): Os objetos MIB são representados com uma linguagem de dados chamada Estrutura de Gestão Informações (SMI). A estruturação é em formato de árvore que contém os objetos gerenciados, cada objeto possui uma identificação única denominada Object Identification (OID), composta por uma sequência de números que identifica a posição do objeto na árvore do MIB. 


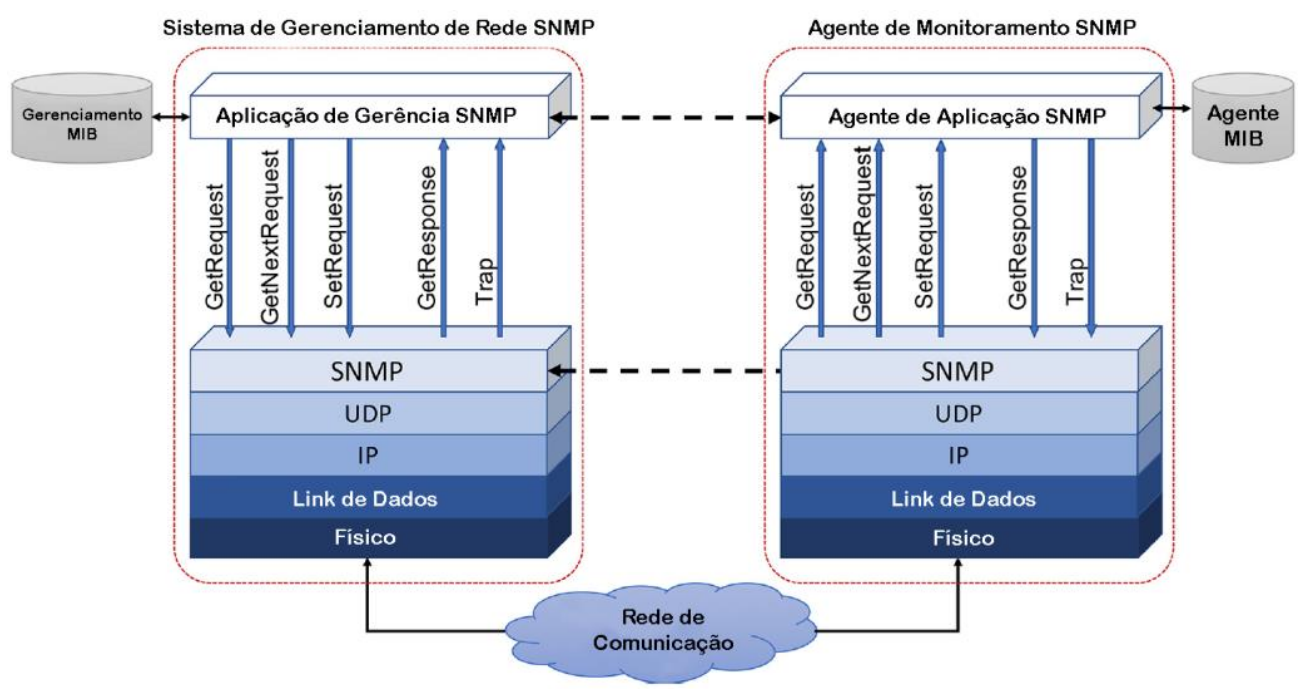

Figura 2. Arquitetura de gerenciamento de redes SNMP [Sinche et al. 2020] (Adaptado pelo Autor).

O protocolo SNMP possui quatro operações comuns entre todas as versões, são elas [Boyko et al. 2019]:

a. Get: Usado pelo gerente para buscar o valor de um objeto em um agente.

b. GetNext: Busca o próximo objeto instanciado pelo Get.

c. Set: Inicia ou reinicia os valores dos objetos instanciados no agente.

d. Trap: É uma mensagem não solicitada pelo gerente, normalmente sobre um evento significante ou falha no sistema monitorado.

\section{Proposta de Desenvolvimento e Metodologia}

Conforme abordado anteriormente, o objetivo deste trabalho é o desenvolvimento de um agente SNMP para ambientes industriais e com o primeiro desenvolvimento voltado a monitoramento de data center. Os data centers são peças chaves no desempenho de uma empresa, idealmente os ativos são mantidos com uma alimentação de energia e resfriamento estável. Como os equipamentos que fazem parte da infraestrutura central da rede são sensíveis, é necessária uma infraestrutura dedicada ao monitoramento de energia, temperatura, umidade e entre outros pontos críticos ao ambiente e sua ininterrupção.

Em empresas que trabalham em $24 / 7$ e não possuem gerência de infraestrutura disponível em todos os horários, a necessidade de monitoramento constante é um fator crucial para o desempenho adequado do data center. Pois em casos de alterações bruscas, como por exemplo, na temperatura do ambiente, pode levar a uma reação em cadeia de danos em equipamentos e paralisação parcial ou total dos serviços e servidores. Estas paralisações podem acarretar em prejuízos enormes e/ou irreversíveis para a empresa, dependendo da magnitude do sinistro ocorrido. Desta forma, proporcionando um sistema de monitoramento adequado, é possível prevenir paradas e mitigar os riscos de perdas mais agressivas. 
O experimento será efetuado em um ambiente de data center corporativo, na Figura 3 é demonstrado o layout da disposição dos componentes presentes. Nestes ambientes, é desejável que se mantenha o monitoramento das variáveis de temperatura, umidade e de gás/incêndio. Para o desenvolvimento, será instalado o sensoriamento no corredor frio em frente ao rack de servidores, pois os mesmos são equipamentos mais sensíveis em comparação com equipamentos de telecomunicações e rede.

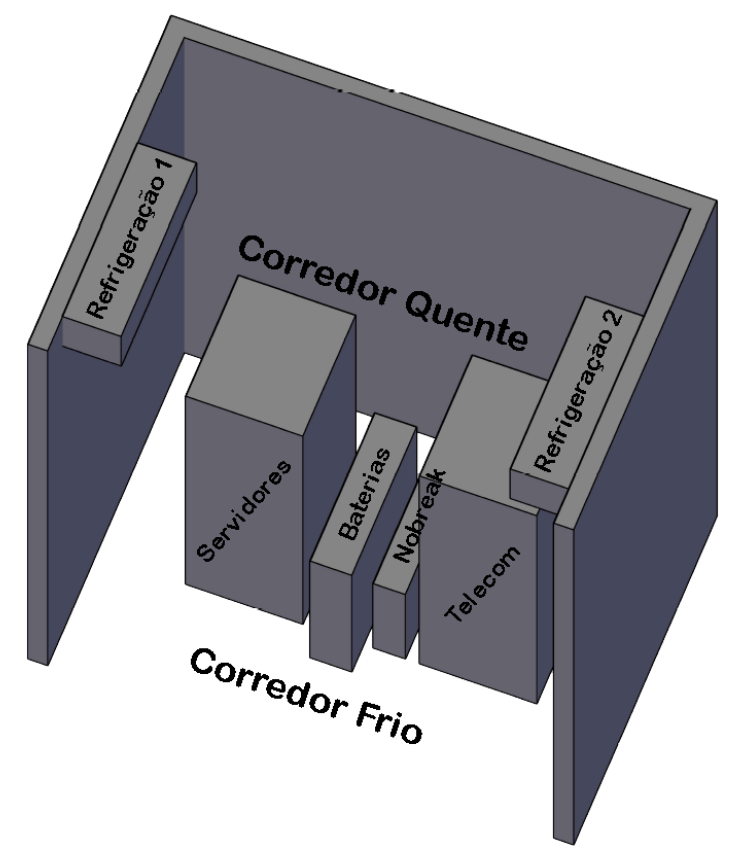

Figura 3. Layout do Data Center.

A estrutura do experimento seguirá as seguintes etapas:

a. Escolha do microcontrolador: Estudo e análise dos microcontroladores capazes de embarcar o protocolo SNMP e possuir comunicação cabeada e/ou Wireless.

b.Escolha dos sensores: Análise do sensoriamento a ser embarcado, levando em consideração as precisões e mantendo o sistema com baixo consumo e processamento necessário baixo.

c. Desenvolvimento do Firmware: Desenvolvimento do firmware necessário para o sensoriamento, criação e configuração do sistema MIB novo no microcontrolador.

d. Testes e validações: Estabilidade do firmware, testes de variações induzidas e temporais sobre os sensores, validação dos parâmetros para acionamentos das Traps e validações da geração corretas a partir do cabeçalho pré-definido do MIB entre agente e gerente.

\section{Resultados Esperados}

Após a finalização das validações em laboratório, será implementado o agente no ambiente definido. Com o auxílio de um software que efetuará o papel de gerente, se busca alcançar um sistema com monitoramento em tempo real, de acordo com as 
informações provenientes do sensoriamento implementado. Configuração de traps no agente, para que em caso de anomalias no ambiente monitorado, o gerente possa prosseguir com os alertas e controles necessários. Com o sistema de forma estável e ininterrupta, será proporcionado uma vida útil maior para os ativos que estão no ambiente. Com base nas informações obtidas, uma análise temporal das variações nas aquisições e a partir das mesmas auxiliar na tomada de decisões.

Para trabalhos posteriores, podem ser efetuadas análises em qualquer ambiente da manufatura e efetuar a busca dos parâmetros que serão monitorados, para isso, somente sendo necessária a troca do sensoriamento, pois todo o restante do sistema está configurado e validado. Com isso, a criação de um agente SNMP modular ajustável a necessidade empresarial do momento.

\section{Referências}

A. Boyko, V. Varkentin and T. Polyakova, "Advantages and Disadvantages of the Data Collection's Method Using SNMP," 2019 International Multi-Conference on Industrial Engineering and Modern Technologies (FarEastCon), Vladivostok, Russia, 2019, pp. 1-5, doi: 10.1109/FarEastCon.2019.8934069.

J. de C. Silva, P. H. M. Pereira, L. L. de Souza, C. N. M. Marins, G. A. B. Marcondes and J. J. P. C. Rodrigues, "Performance Evaluation of IoT Network Management Platforms," 2018 International Conference on Advances in Computing, Communications and Informatics (ICACCI), Bangalore, 2018, pp. 259-265, doi: 10.1109/ICACCI.2018.8554364.

Kagermann, H.; Wahlster, W.; Helbig, J. (2013). "Recommendations for implementing the strategic initiative INDUSTRIE 4.0”. ACATECH.

S. Sinche et al., "A Survey of IoT Management Protocols and Frameworks," in IEEE Communications Surveys \& Tutorials, vol. 22, no. 2, pp. 1168-1190, Secondquarter 2020, doi: 10.1109/COMST.2019.2943087.

Santos, F. Sérgio dos (2017). "Aplicação do protocolo SNMP para o monitoramento on line de uma microgeração fotovoltaica”, https://repositorio.unesp.br/ handle/11449/150972, Dezembro.

Schneider, J. (2018). "Medição do nível de maturidade do uso de tecnologia em um ambiente da indústria 4.0", https://repositorio.ucs.br/11338/4877, Dezembro.

Silva, J. d. C. (2018). "Performance assessment of management protocols and platforms for internet of things", http://tede.inatel.br:8080/jspui/handle/tede/182, Dezembro.

Machado, L. F. (2015). "Proxy IP de baixo custo e múltiplos sensores para cidades inteligentes", http://tede.bibliotecadigital.puc-campinas.edu.br:8080/jspui//handle/ tede/560, Dezembro.

W. T. Poloni, R. Becker and R. Balbinot, "Remote control of low cost devices using SNMP agents," 2017 IEEE Pacific Rim Conference on Communications, Computers and Signal Processing (PACRIM), Victoria, BC, 2017, pp. 1-6, doi: 10.1109/PACRIM.2017.8121927. 